MATHEMATICS OF COMPUTATION

Volume 72, Number 241, Pages 419-433

S 0025-5718(02)01433-3

Article electronically published on May 1, 2002

\title{
A CONVERGENCE AND STABILITY STUDY OF THE ITERATED LUBKIN TRANSFORMATION AND THE $\theta$-ALGORITHM
}

\author{
AVRAM SIDI
}

\begin{abstract}
In this paper we analyze the convergence and stability of the iterated Lubkin transformation and the $\theta$-algorithm as these are being applied to sequences $\left\{A_{n}\right\}$ whose members behave like $A_{n} \sim A+\zeta^{n} /(n !)^{r} \sum_{i=0}^{\infty} \alpha_{i} n^{\gamma-i}$ as $n \rightarrow \infty$, where $\zeta$ and $\gamma$ are complex scalars and $r$ is a nonnegative integer. We study the three different cases in which (i) $r=0, \zeta=1$, and $\gamma \neq 0,1, \ldots$ (logarithmic sequences), (ii) $r=0$ and $\zeta \neq 1$ (linear sequences), and (iii) $r=1,2, \ldots$ (factorial sequences). We show that both methods accelerate the convergence of all three types of sequences. We show also that both methods are stable on linear and factorial sequences, and they are unstable on logarithmic sequences. On the basis of this analysis we propose ways of improving accuracy and stability in problematic cases. Finally, we provide a comparison of these results with analogous results corresponding to the Levin $u$-transformation.
\end{abstract}

\section{INTRODUCTION AND BACKGROUND}

The purpose of this work is to contribute to our understanding of how the iterated $W$-transformation of Lubkin [7] and the $\theta$-algorithm of Brezinski [2] accelerate the convergence of some important classes of infinite sequences $\left\{A_{n}\right\}$. The sequences that we have in mind are the following:

1. Logarithmic sequences for which

$$
A_{n} \sim A+\sum_{i=0}^{\infty} \alpha_{i} n^{\gamma-i} \text { as } n \rightarrow \infty ; \alpha_{0} \neq 0, \gamma \neq 0,1,2, \ldots \text {. }
$$

Here $A=\lim _{n \rightarrow \infty} A_{n}$ when $\Re \gamma<0$. When $\Re \gamma \geq 0, A$ is the antilimit of $\left\{A_{n}\right\}$.

2. Linear sequences for which

$$
A_{n} \sim A+\zeta^{n} \sum_{i=0}^{\infty} \alpha_{i} n^{\gamma-i} \text { as } n \rightarrow \infty ; \alpha_{0} \neq 0, \zeta \neq 1 .
$$

Here $A=\lim _{n \rightarrow \infty} A_{n}$ when (a) $|\zeta|<1$ or (b) $|\zeta|=1$ and $\Re \gamma<0$. Otherwise, $A$ is the antilimit of $\left\{A_{n}\right\}$.

Received by the editor March 21, 2001.

2000 Mathematics Subject Classification. Primary 65B05, 65B10, 40A05, 40A25, 41A60.

(C)2002 American Mathematical Society 
3. Factorial sequences for which

$$
A_{n} \sim A+\frac{\zeta^{n}}{(n !)^{r}} \sum_{i=0}^{\infty} \alpha_{i} n^{\gamma-i} \text { as } n \rightarrow \infty ; \alpha_{0} \neq 0, r=1,2, \ldots
$$

Here $A=\lim _{n \rightarrow \infty} A_{n}$ always.

Of these, the classes of logarithmic and linear sequences have provided researchers with important test grounds for the comparative study of convergence acceleration methods, including the Shanks transformation [9] (or the equivalent $\varepsilon$-algorithm of Wynn [18]), the $\theta$-algorithm, the iterated $W$-transformation, the Levin [5] transformations, and so on. See, e.g., Smith and Ford [15, 16] and Van Tuyl [17].

If we let $a_{0}=A_{0}$ and $a_{n}=\Delta A_{n-1}=A_{n}-A_{n-1}, n=1,2, \ldots$, then we realize that the $a_{n}$ grow as in

$$
\left.a_{n} \sim \sum_{i=0}^{\infty} e_{i} n^{\gamma-1-i} \text { as } n \rightarrow \infty ; \quad e_{0}=\gamma \alpha_{0} \neq 0, \gamma \neq 0,1, \ldots \quad \text { (for (1.1) }\right) \text {, }
$$

$$
\left.a_{n} \sim \zeta^{n} \sum_{i=0}^{\infty} e_{i} n^{\gamma-i} \text { as } n \rightarrow \infty ; \quad e_{0}=\left(1-\zeta^{-1}\right) \alpha_{0} \neq 0, \zeta \neq 1 \quad \text { (for [1.2) }\right),
$$

$$
\left.a_{n} \sim \frac{\zeta^{n}}{(n !)^{r}} \sum_{i=0}^{\infty} e_{i} n^{\gamma+r-i} \text { as } n \rightarrow \infty ; \quad e_{0}=-\zeta^{-1} \alpha_{0} \neq 0, r=1,2, \ldots \quad \text { (for (11.3) }\right)
$$

(Of course, the $e_{i}$ are different in each of (1.4)-(1.6).) Furthermore, the $a_{n}$ satisfy 2 -term recursion relations of the form

$$
a_{n+1}=c(n) a_{n},
$$

where $c(n)$ satisfies asymptotically

$$
\left.c(n) \sim 1+(\gamma-1) n^{-1}+c_{2} n^{-2}+\cdots \quad \text { as } n \rightarrow \infty ; \quad \gamma \neq 0,1,2, \ldots \text { (in (1.1) }\right),
$$

$$
c(n) \sim \zeta\left(1+\gamma n^{-1}+c_{2} n^{-2}+\cdots\right) \text { as } n \rightarrow \infty ; \quad \zeta \neq 1 \quad(\text { in }(1.2)),
$$

$$
\left.c(n) \sim \zeta n^{-r}\left(1+\gamma n^{-1}+c_{2} n^{-2}+\cdots\right) \quad \text { as } n \rightarrow \infty ; \quad r=1,2, \ldots \quad \text { (in (1.3) }\right),
$$

Conversely, if the $a_{n}$ satisfy (1.7) with (1.8) or (1.9) or (1.10), then they have asymptotic expansions that are given exactly as in (1.4) or (1.5) or (1.6), respectively. This has been shown in Ford [4, p. 70].

Conversely again, if $a_{n}$ are as in (1.4) or (1.5) or (1.6) and $A_{n}=\sum_{k=0}^{n} a_{k}$, $n=0,1, \ldots$, then the $A_{n}$ have asymptotic expansions that are given exactly as in 
(1.1) or (1.2) or (1.3), respectively. This follows from the following results:

$$
\begin{aligned}
& A_{n} \sim A+n a_{n+1} \sum_{i=0}^{\infty} \beta_{i} n^{-i} \text { as } n \rightarrow \infty ; \quad \beta_{0}=\gamma^{-1} \neq 0 \text { for (1.4) } \\
& A_{n} \sim A+a_{n+1} \sum_{i=0}^{\infty} \beta_{i} n^{-i} \text { as } n \rightarrow \infty ; \beta_{0}=(\zeta-1)^{-1} \neq 0 \text { for (1.5), } \\
& A_{n} \sim A+a_{n+1}\left(-1+\sum_{i=r}^{\infty} \beta_{i} n^{-i}\right) \text { as } n \rightarrow \infty ; \quad \beta_{r}=-\zeta \neq 0 \text { for (1.6). }
\end{aligned}
$$

The expansions in (1.11) and (1.12) were given by the author in [10] and [11] for convergent $\sum_{k=0}^{\infty} a_{k}$. The result in (1.11) for divergent $\sum_{k=0}^{\infty} a_{k}$ was given in [13, where the nature of the antilimit $A$ and its precise value are also provided. The result in (1.12) for divergent $\sum_{k=0}^{\infty} a_{k}$ was given in [12] for the case $|\zeta|=1, \zeta \neq 1$, where it is shown that the antilimit $A$ is the Abel sum of $\sum_{k=0}^{\infty} a_{k}$, namely, $A=$ $\lim _{\tau \rightarrow 1-} \sum_{k=0}^{\infty} a_{k} \tau^{k}$. $A$ is also the analytic continuation to $|\zeta|=1$ of $f(\zeta)$, the function that is defined by the power series $\sum_{k=0}^{\infty} c_{k} \zeta^{k}, c_{n}=a_{n} \zeta^{-n}, n=0,1, \ldots$, and that is analytic for $|\zeta|<1$. In [12] it is shown in addition that (1.12) holds also when $|\zeta|>1, \zeta \notin[1,+\infty)$, if $a_{n}=\zeta^{n} n^{p} h(n)$, where $p$ is a nonnegative integer and $h(n)=\int_{0}^{\infty} e^{-n t} \varphi(t) d t, \varphi(t)$ being a function of exponential order for which $a_{n}$ has an asymptotic expansion of the form given in (1.5). In this case $A$, the antilimit of $\left\{A_{n}\right\}$, is $f(\zeta)$, described above, that is now defined and analytic for all $\zeta$ in the complex plane cut along $[1,+\infty)$.

The result in (1.13) is new and can be proved as follows: Since $\left\{A_{n}\right\}$ converges when $a_{n}$ is as in (1.6), we have $A_{n}-A=-\sum_{k=n+1}^{\infty} a_{k}$ and $\sum_{k=n+1}^{\infty} a_{k}$ is a convergent series. Invoking (1.7), we can write

$$
A_{n}-A=-a_{n+1}\left[1+\sum_{k=1}^{\infty} \prod_{j=1}^{k} c(n+j)\right] \text {. }
$$

By (1.10) the term inside the square brackets has an asymptotic expansion of the form $1+\sum_{i=r}^{\infty} \sigma_{i} n^{-i}$ as $n \rightarrow \infty$, with $\sigma_{r}=\zeta$. This produces (1.13). (We must note, however, that an expansion of the form $A_{n} \sim A+a_{n+1} \sum_{i=0}^{\infty} \beta_{i} n^{-i}$ as $n \rightarrow \infty$ is already contained in the paper [10] for the case in which $\left\{A_{n}\right\}$ satisfies (1.3).)

The results in (1.11)-(1.13) will be used later in proving theorems on the convergence and convergence acceleration of the (iterated) $W$-transformation and the $\theta$-algorithm. It must be noted here that the fact that $\beta_{1}=\cdots=\beta_{r-1}=0$ in (1.13) is of great importance in the convergence theorems of the next two sections. Without this knowledge one cannot show that there is convergence acceleration, for example.

We now turn to the definitions of the $W$ - and iterated $W$-transformations and the $\theta$-algorithm.

Two convenient representations of the $W$-transformation on $\left\{A_{k}\right\}$ are given by

$$
W_{j}\left(\left\{A_{s}\right\}\right)=\frac{\Delta^{2}\left(A_{j} / \Delta A_{j}\right)}{\Delta^{2}\left(1 / \Delta A_{j}\right)}=\frac{\Delta\left(A_{j+1} \times \Delta\left(1 / \Delta A_{j}\right)\right)}{\Delta^{2}\left(1 / \Delta A_{j}\right)}, j=0,1, \ldots
$$


This transformation is normally applied in iterated form as in

$$
\begin{aligned}
B_{0}^{(j)} & =A_{j}, \quad j=0,1, \ldots, \\
B_{n+1}^{(j)} & =W_{j}\left(\left\{B_{n}^{(s)}\right\}\right), \quad j, n=0,1, \ldots .
\end{aligned}
$$

Thus $B_{1}^{(j)}=W_{j}\left(\left\{A_{s}\right\}\right)$. In addition, $B_{n}^{(j)}$ is determined from $A_{j+k}, k=$ $0,1, \ldots, 3 n$.

The $\theta$-algorithm on $\left\{A_{k}\right\}$ is defined via the recursion relations

$$
\begin{aligned}
& \theta_{-1}^{(j)}=0, \quad \theta_{0}^{(j)}=A_{j}, \quad j=0,1, \ldots, \\
& \theta_{2 n+1}^{(j)}=\theta_{2 n-1}^{(j+1)}+\frac{1}{\Delta \theta_{2 n}^{(j)}}, \\
& \theta_{2 n+2}^{(j)}=\theta_{2 n}^{(j+1)}+\frac{\Delta \theta_{2 n}^{(j+1)} \times \Delta \theta_{2 n+1}^{(j+1)}}{\Delta^{2} \theta_{2 n+1}^{(j)}}, \quad j, n=0,1, \ldots,
\end{aligned}
$$

where $\Delta \theta_{p}^{(j)}=\theta_{p}^{(j+1)}-\theta_{p}^{(j)}$ for all $j$ and $p$.

Note that $\theta_{2 n}^{(j)}$ is determined from $A_{j+k}, k=0,1, \ldots, 3 n$, just like $B_{n}^{(j)}$.

Using the fact that $\theta_{1}^{(j)}=1 / \Delta A_{j}$ in (1.16), it can be shown after some algebra that

$$
\theta_{2}^{(j)}=W_{j}\left(\left\{A_{s}\right\}\right) .
$$

This fact was first noted by Drummond [3]. Also, from the fact that the Levin $u$-transformation on $\left\{A_{k}\right\}$ produces the approximations $u_{n}^{(j)}$ to $A$ as in

$$
u_{n}^{(j)}=\frac{\Delta^{n}\left(j^{n-2} A_{j} / \Delta A_{j}\right)}{\Delta^{n}\left(j^{n-2} / \Delta A_{j}\right)},
$$

see Sidi [10], it is clear that

$$
u_{2}^{(j)}=W_{j}\left(\left\{A_{s}\right\}\right),
$$

and this was first observed by Bhowmick, Bhattacharya, and Roy 1 .

Sablonnière [8] and Van Tuyl [17] have provided the convergence analysis for the sequences $\left\{B_{n}^{(j)}\right\}_{j=0}^{\infty}$ as these are obtained from logarithmic sequences $\left\{A_{k}\right\}$ in (1.1). (In fact, both papers deal with sequences $\left\{A_{k}\right\}$ for which $A_{n} \sim A+\sum_{i=0}^{\infty} \alpha_{i} n^{\gamma-i / s}$ as $n \rightarrow \infty$, where $s=1,2, \ldots$. In [8] explicit treatment is given for $s=2$, while in [17] $s$ is any integer.) The convergence analysis of the sequences $\left\{\theta_{2 n}^{(j)}\right\}_{j=0}^{\infty}$ from logarithmic sequences $\left\{A_{k}\right\}$ has been given in [17]. We are not aware of analogous studies pertaining to the linear and factorial sequences in (1.2) and (1.3). Furthermore, no results on the stability of the sequences $\left\{B_{n}^{(j)}\right\}_{j=0}^{\infty}$ and $\left\{\theta_{2 n}^{(j)}\right\}_{j=0}^{\infty}$ have been obtained so far. Our purpose in the present work is to provide these missing analyses. We give the convergence and stability theories of the sequences $\left\{B_{n}^{(j)}\right\}_{j=0}^{\infty}$ and $\left\{\theta_{2 n}^{(j)}\right\}_{j=0}^{\infty}$ as these are obtained from the logarithmic, linear, and factorial sequences $\left\{A_{k}\right\}$ given in 1.1 - 1.3). A nice feature of our analysis is that the treatments of the three fundamentally different classes (linear, logarithmic, factorial) are unified and simplified. In addition, based on the stability analyses of $\left\{B_{n}^{(j)}\right\}_{j=0}^{\infty}$ and $\left\{\theta_{2 n}^{(j)}\right\}_{j=0}^{\infty}$ from linear sequences $\left\{A_{k}\right\}$, we are able to conclude that the strategy proposed in [12] for power series and Fourier series near points of singularity can be applied very effectively here too. All this is done in Sections 
24. The study of the convergence of the $W$-transformation that is the subject of Section 2 turns out to be of great importance in the developments of Sections 3 and 4 ,

Finally, in Section 5 we provide a detailed comparison of our results on the iterated Lubkin transformation and the $\theta$-algorithm with the corresponding results pertaining to the Levin $u$-transformation.

\section{Convergence analysis of the iterated Lubkin transformation}

We start with the $W$-transformation of (1.14).

Theorem 2.1. (i) Let $\left\{A_{k}\right\}$ be as in (1.1), and let $\beta_{2+\mu}(\mu \geq 0)$ be the first nonzero $\beta_{i}$ with $i \geq 2$ in (1.11). Then

$$
W_{j}\left(\left\{A_{s}\right\}\right)-A \sim \sum_{i=0}^{\infty} w_{i} j^{\gamma-2-\mu-i} \text { as } j \rightarrow \infty ; \quad w_{0}=\alpha_{0} \beta_{2+\mu} \frac{(\mu+1)(\mu+2)}{\gamma-1} \neq 0 .
$$

(ii) Let $\left\{A_{k}\right\}$ be as in $\left[1.2\right.$, and let $\beta_{1+\mu}(\mu \geq 0)$ be the first nonzero $\beta_{i}$ with $i \geq 1$ in (1.12). Then

$$
W_{j}\left(\left\{A_{s}\right\}\right)-A \sim \zeta^{j} \sum_{i=0}^{\infty} w_{i} j^{\gamma-3-\mu-i} \text { as } j \rightarrow \infty ; \quad w_{0}=\alpha_{0} \beta_{1+\mu} \frac{(\mu+1)(\mu+2)}{\zeta-1} \zeta^{2} \neq 0 .
$$

(iii) Let $\left\{A_{k}\right\}$ be as in 1.3 . Then

$$
W_{j}\left(\left\{A_{s}\right\}\right)-A \sim \frac{\zeta^{j}}{(j !)^{r}} \sum_{i=0}^{\infty} w_{i} j^{\gamma-3 r-2-i} \text { as } j \rightarrow \infty ; \quad w_{0}=\alpha_{0} \zeta^{3} r(r+1) \neq 0 .
$$

Part (i) of Theorem 2.1 was already given in [7]. All three of (2.1)-(2.3) also follow from the analogous results of [10] and [11] that pertain to the Levin transformations. They can be proved by observing that

$$
W_{j}\left(\left\{A_{s}\right\}\right)-A=\frac{\Delta^{2}\left(\left(A_{j}-A\right) / \Delta A_{j}\right)}{\Delta^{2}\left(1 / \Delta A_{j}\right)},
$$

and by invoking (1.11)-(1.13) and (1.4)-(1.6). In addition, we need the following facts that we will use again later in this work: Let

$$
g(n) \sim \sum_{i=0}^{\infty} g_{i} n^{\delta-i} \text { as } n \rightarrow \infty ; \quad g_{0} \neq 0 .
$$

First, if $\delta \neq 0$,

$$
\Delta g(n) \sim \sum_{i=0}^{\infty} \hat{g}_{i} n^{\delta-i-1} \text { as } n \rightarrow \infty ; \quad \hat{g}_{0}=\delta g_{0} \neq 0,
$$

and if $\delta=0$ and $g_{\nu}$ is the first nonzero $g_{i}$ with $i \geq 1$,

$$
\Delta g(n) \sim \sum_{i=\nu}^{\infty} \hat{g}_{i} n^{-i-1} \text { as } n \rightarrow \infty ; \quad \hat{g}_{\nu}=-\nu g_{\nu} \neq 0 .
$$


Next, if $\zeta \neq 1$,

$$
\Delta\left[\zeta^{n} g(n)\right] \sim \zeta^{n} \sum_{i=0}^{\infty} \hat{g}_{i} n^{\delta-i} \text { as } n \rightarrow \infty ; \quad \hat{g}_{0}=(\zeta-1) g_{0} \neq 0 .
$$

Finally, if $p=1,2, \ldots$,

$$
\begin{aligned}
& \Delta\left[(n !)^{p} \zeta^{n} g(n)\right] \sim(n !)^{p} \zeta^{n} \sum_{i=0}^{\infty} \hat{g}_{i} n^{\delta+p-i} \text { as } n \rightarrow \infty ; \quad \hat{g}_{0}=\zeta g_{0} \neq 0 . \\
& \Delta\left[\frac{\zeta^{n}}{(n !)^{p}} g(n)\right] \sim \frac{\zeta^{n}}{(n !)^{p}} \sum_{i=0}^{\infty} \hat{g}_{i} n^{\delta-i} \text { as } n \rightarrow \infty ; \quad \hat{g}_{0}=-g_{0} .
\end{aligned}
$$

It follows from Theorem 2.1 that the $W$-transformation accelerates the convergence of logarithmic, linear, and factorial sequences, the acceleration of linear sequences being more effective in general. Specifically,

$$
\begin{aligned}
& \frac{W_{j}\left(\left\{A_{s}\right\}\right)-A}{A_{j+i}-A}=O\left(j^{-2}\right) \text { as } j \rightarrow \infty, \quad i \text { fixed (in (1.1)), } \\
& \frac{W_{j}\left(\left\{A_{s}\right\}\right)-A}{A_{j+i}-A}=O\left(j^{-3}\right) \text { as } j \rightarrow \infty, \quad i \text { fixed (in (1.2)), } \\
& \frac{W_{j}\left(\left\{A_{s}\right\}\right)-A}{A_{j+3}-A} \sim r(r+1) j^{-2} \text { as } j \rightarrow \infty, \quad \text { in (1.3)). }
\end{aligned}
$$

Note that since $W_{j}\left(\left\{A_{s}\right\}\right)$ is constructed from $A_{j+i}, 0 \leq i \leq 3$, we should compare $W_{j}\left(\left\{A_{s}\right\}\right)-A$ with $A_{j+i}-A, 0 \leq i \leq 3$. When $\left\{A_{k}\right\}$ is as in (1.1) or (1.2), $A_{j+i}-A$ are all of the same order with arbitrary fixed $i$. When $\left\{A_{k}\right\}$ is as in (1.3), however, $W_{j}\left(\left\{A_{s}\right\}\right)-A$ must be compared with $A_{j+3}-A$, the smallest of $A_{j+i}-A, 0 \leq i \leq 3$, as $j \rightarrow \infty$, and this is what we have done in (2.13).

Finally, from (2.1) it is clear that, whether $\left\{A_{k}\right\}$ in (1.1) converges or not, $W_{j}\left(\left\{A_{s}\right\}\right)$ converges to $A$ if $\Re \gamma<2$. Similarly, from (2.2) we see that $W_{j}\left(\left\{A_{s}\right\}\right)$ converges to $A$ when $|\zeta|=1$ provided $\Re \gamma<3$, whether $\left\{A_{k}\right\}$ in (1.2) converges or not.

The following theorem concerns the repeated $W$-transformation as defined in (1.15) and can be proved by repeated application of Theorem 2.1. This is possible since the asymptotic expansion of $W_{j}\left(\left\{A_{s}\right\}\right)$ as $j \rightarrow \infty$ in Theorem 2.1 is of precisely the same nature as that of $A_{j}$.

Theorem 2.2. (i) If $\left\{A_{k}\right\}$ is as in (1.1), then

$$
B_{n}^{(j)}-A \sim \sum_{i=0}^{\infty} g_{n i} j^{\gamma_{n}-i} \text { as } j \rightarrow \infty, \quad g_{n 0} \neq 0,
$$

with $\gamma_{0}=\gamma, \gamma_{k}=\gamma_{k-1}-2-\mu_{k}, k=1,2, \ldots$, where $\mu_{k}$ are some nonnegative integers, hence $\Re \gamma_{k} \leq \Re \gamma-2 k, k=1,2, \ldots$.

(ii) If $\left\{A_{k}\right\}$ is as in (1.2), then

$$
B_{n}^{(j)}-A \sim \zeta^{j} \sum_{i=0}^{\infty} g_{n i} j^{\gamma_{n}-i} \text { as } j \rightarrow \infty, \quad g_{n 0} \neq 0,
$$

with $\gamma_{0}=0, \gamma_{k}=\gamma_{k-1}-3-\mu_{k}, k=1,2, \ldots$, where $\mu_{k}$ are some nonnegative integers, hence $\Re \gamma_{k} \leq \Re \gamma-3 k, k=1,2, \ldots$. 
(iii) If $\left\{A_{k}\right\}$ is as in (1.3), then

$$
B_{n}^{(j)}-A \sim \frac{\zeta^{j}}{(j !)^{r}} \sum_{i=0}^{\infty} g_{n i} j^{\gamma_{n}-i} \text { as } j \rightarrow \infty, \quad g_{n 0}=\alpha_{0}\left[\zeta^{3} r(r+1)\right]^{n} \neq 0,
$$

with $\gamma_{k}=\gamma-k(3 r+2), k=0,1, \ldots$.

Part (i) of Theorem [2.1] was already given in [8] and [17]. Parts (ii) and (iii) are new.

Clearly, the repeated $W$-transformation accelerates the convergence of logarithmic, linear, and factorial sequences. Even when $\Re \gamma \geq 0$, hence $\left\{A_{k}\right\}$ in (1.1) diverges, $\left\{B_{n}^{(j)}\right\}_{j=0}^{\infty}$ will converge to $A$ for every $n>\frac{1}{2} \Re \gamma$ in part (i) of Theorem 2.2. Similarly, when $\left\{A_{k}\right\}$ is as in (1.2) with $|\zeta|=1,\left\{B_{n}^{(j)}\right\}_{j=0}^{\infty}$ will converge to $A$ for every $n>\frac{1}{3} \Re \gamma$ in part (ii) of Theorem 2.2, even when $\left\{A_{k}\right\}$ diverges.

In addition, analogously to (2.11) -(2.13), we have

$$
\begin{aligned}
& \left.\frac{B_{n+1}^{(j)}-A}{B_{n}^{(j+i)}-A}=O\left(j^{-2}\right) \text { as } j \rightarrow \infty, i \text { fixed (in (1.1) }\right) \text {, } \\
& \left.\frac{B_{n+1}^{(j)}-A}{B_{n}^{(j+i)}-A}=O\left(j^{-3}\right) \text { as } j \rightarrow \infty, i \text { fixed (in (1.2) }\right) \text {, } \\
& \frac{B_{n+1}^{(j)}-A}{B_{n}^{(j+3)}-A} \sim r(r+1) j^{-2} \text { as } j \rightarrow \infty \quad(\text { in (1.3) }) \text {. }
\end{aligned}
$$

In other words, the sequence $\left\{B_{n+1}^{(j)}\right\}_{j=0}^{\infty}$ converges more quickly than $\left\{B_{n}^{(j)}\right\}_{j=0}^{\infty}$ for each $n$ in all cases.

\section{Convergence analysis of the $\theta$-Algorithm}

Due to the complexity of the recursion relation in (1.16), the analysis of the $\theta$-algorithm turns out to be quite involved. Specifically, we have two different types of sequences to worry about, namely, $\left\{\theta_{2 n}^{(j)}\right\}_{j=0}^{\infty}$ and $\left\{\theta_{2 n+1}^{(j)}\right\}_{j=0}^{\infty}$, and the two are coupled nonlinearly. Luckily, a very rigorous analysis of both types of sequences can be given, and we turn to it now.

We start by expressing $\theta_{2 n+2}^{(j)}$ in (11.16) in different forms.

Lemma 3.1. $\theta_{2 n+2}^{(j)}$ can be expressed as in

$$
\theta_{2 n+2}^{(j)}=\frac{1}{\Delta^{2} \theta_{2 n+1}^{(j)}}\left\{W_{j}\left(\left\{\theta_{2 n}^{(s)}\right\}\right) \times \Delta^{2}\left(1 / \Delta \theta_{2 n}^{(j)}\right)+\Delta\left(\theta_{2 n}^{(j+1)} \times \Delta \theta_{2 n-1}^{(j+1)}\right)\right\}
$$

and

$$
\theta_{2 n+2}^{(j)}=\frac{\Delta\left(\theta_{2 n}^{(j+1)} \times \Delta \theta_{2 n+1}^{(j)}\right)}{\Delta^{2} \theta_{2 n+1}^{(j)}} .
$$

Proof. From (1.16) we first have

$$
\theta_{2 n+2}^{(j)}=\frac{\theta_{2 n}^{(j+1)} \times \Delta^{2} \theta_{2 n+1}^{(j)}+\Delta \theta_{2 n}^{(j+1)} \times \Delta \theta_{2 n+1}^{(j+1)}}{\Delta^{2} \theta_{2 n+1}^{(j)}} .
$$


Substituting $\Delta \theta_{2 n}^{(j+1)}=\theta_{2 n}^{(j+2)}-\theta_{2 n}^{(j+1)}$ and $\Delta^{2} \theta_{2 n+1}^{(j)}=\Delta \theta_{2 n+1}^{(j+1)}-\Delta \theta_{2 n+1}^{(j)}$ in the numerator of (3.3), we obtain (3.2). Next, substituting $\theta_{2 n+1}^{(j)}=\theta_{2 n-1}^{(j+1)}+1 / \Delta \theta_{2 n}^{(j)}$ in the numerator of (3.3), we have

$$
\theta_{2 n+2}^{(j)}=\frac{\Delta\left(\theta_{2 n}^{(j+1)} \times \Delta\left(1 / \Delta \theta_{2 n}^{(j)}\right)\right)+\Delta\left(\theta_{2 n}^{(j+1)} \times \Delta \theta_{2 n-1}^{(j+1)}\right)}{\Delta^{2} \theta_{2 n+1}^{(j)}} .
$$

Letting now $n=0$ and recalling that $\theta_{-1}^{(j)}=0, \theta_{0}^{(j)}=A_{j}$, and hence $\theta_{1}^{(j)}=1 / \Delta A_{j}$, we have from (3.4) and (1.14) that

$$
\theta_{2}^{(j)}=\frac{\Delta\left(A_{j+1} \times \Delta\left(1 / \Delta A_{j}\right)\right)}{\Delta^{2}\left(1 / \Delta A_{j}\right)}=W_{j}\left(\left\{A_{s}\right\}\right) .
$$

(This is nothing but (1.17).) Using this in (3.4), the result in (3.1) follows.

The representation of $\theta_{2 n+2}^{(j)}$ given in (3.1) of Lemma3.1 and Theorem 2.1 enable us to analyze in a unified manner the convergence of the sequences $\left\{\theta_{2 n}^{(j)}\right\}_{j=0}^{\infty}$ for all three cases of $\left\{A_{n}\right\}$ described in (1.1) (1.3).

Theorem 3.2. (i) If $\left\{A_{k}\right\}$ is as in (1.1), then

$$
\begin{aligned}
\theta_{2 n}^{(j)}-A & \sim \sum_{i=0}^{\infty} g_{n i} j^{\gamma_{n}-i} \text { as } j \rightarrow \infty, \quad g_{n 0} \neq 0, \\
\theta_{2 n+1}^{(j)} & \sim \sum_{i=0}^{\infty} h_{n i} j^{-\gamma_{n}-i+1} \text { as } j \rightarrow \infty, \quad h_{n 0}=1 /\left(\gamma_{n} g_{n 0}\right) \neq 0,
\end{aligned}
$$

with $\gamma_{0}=\gamma, \gamma_{k}=\gamma_{k-1}-2-\mu_{k}, k=1,2, \ldots$, where $\mu_{k}$ are some nonnegative integers, hence $\Re \gamma_{k} \leq \Re \gamma-2 k, k=1,2, \ldots$.

(ii) If $\left\{A_{k}\right\}$ is as in (1.2), then

$$
\begin{aligned}
\theta_{2 n}^{(j)}-A & \sim \zeta^{j} \sum_{i=0}^{\infty} g_{n i} j^{\gamma_{n}-i} \text { as } j \rightarrow \infty, \quad g_{n 0} \neq 0, \\
\theta_{2 n+1}^{(j)} & \sim \zeta^{-j} \sum_{i=0}^{\infty} h_{n i} j^{-\gamma_{n}-i} \text { as } j \rightarrow \infty, \quad h_{n 0}=1 /\left[(\zeta-1) g_{n 0}\right] \neq 0,
\end{aligned}
$$

with $\gamma_{0}=\gamma, \gamma_{k}=\gamma_{k-1}-3-\mu_{k}, k=1,2, \ldots$, where $\mu_{k}$ are some nonnegative integers, hence $\Re \gamma_{k} \leq \Re \gamma-3 k, k=1,2, \ldots$.

(iii) If $\left\{A_{k}\right\}$ is as in (1.3), then

$$
\begin{gathered}
\theta_{2 n}^{(j)}-A \sim \frac{\zeta^{j}}{(j !)^{r}} \sum_{i=0}^{\infty} g_{n i} j^{\gamma_{n}-i} \text { as } j \rightarrow \infty, \quad g_{n 0}=\alpha_{0}\left[\zeta^{3} r(r+1)\right]^{n} \neq 0, \\
\theta_{2 n+1}^{(j)} \sim \zeta^{-j}(j !)^{r} \sum_{i=0}^{\infty} h_{n i} j^{-\gamma_{n}-i} \text { as } j \rightarrow \infty, \quad h_{n 0}=-1 / g_{n 0} \neq 0,
\end{gathered}
$$

with $\gamma_{0}=\gamma, \gamma_{k}=\gamma-k(3 r+2), k=1,2, \ldots$. Thus, in this case we also have that $\lim _{j \rightarrow \infty}\left[\left(\theta_{2 n}^{(j)}-A\right) /\left(B_{n}^{(j)}-A\right)\right]=1$. 
Remark. Part (i) of this theorem was given in [17]. Parts (ii) and (iii) seem to be new. The $\gamma_{n}$ in (3.6) and (3.8) are not necessarily the same as those in (2.14) and (2.15) respectively.

Proof. Here we shall give a detailed proof of part (ii) of the theorem. The proofs of part (i) and part (iii) can be achieved by following the steps of the proof of part (ii) and by making the appropriate substitutions there.

We proceed by induction on $n$. From the fact that $\theta_{0}^{(j)}=A_{j}$ and by (1.2) it is clear that (3.8) holds for $n=0$ with $\gamma_{0}=\gamma$ and $g_{00}=\alpha_{0} \neq 0$. From this and from the fact that $\theta_{1}^{(j)}=1 / \Delta A_{j}$ it follows that (3.9) holds for $n=0$ with $h_{00}=1 /\left[(\zeta-1) \alpha_{0}\right] \neq 0$. Let us now assume that (3.8) and (3.9) hold, and prove that they hold with $n$ replaced by $n+1$.

We begin by observing that equality is maintained in (1.16) if we subtract $A$ from the $\theta_{2 p}^{(s)}$ there. As a result of this and of Lemma 3.1, we can write

$$
\begin{aligned}
\theta_{2 n+2}^{(j)} & -A \\
& =\frac{1}{\Delta^{2} \theta_{2 n+1}^{(j)}}\left\{\left[W_{j}\left(\left\{\theta_{2 n}^{(s)}\right\}\right)-A\right] \Delta^{2}\left(1 / \Delta \theta_{2 n}^{(j)}\right)+\Delta\left[\left(\theta_{2 n}^{(j+1)}-A\right)\left(\Delta \theta_{2 n-1}^{(j+1)}\right)\right]\right\} .
\end{aligned}
$$

Now by part (ii) of Theorem 2.1 we have

$$
P_{j} \equiv W_{j}\left(\left\{\theta_{2 n}^{(s)}\right\}\right)-A \sim \zeta^{j} \sum_{i=0}^{\infty} p_{i} j^{\gamma_{n}-3-i} \text { as } j \rightarrow \infty,
$$

and

$$
Q_{j} \equiv \Delta^{2}\left(1 / \Delta \theta_{2 n}^{(j)}\right) \sim \zeta^{-j} \sum_{i=0}^{\infty} q_{i} j^{-\gamma_{n}-i} \text { as } j \rightarrow \infty
$$

Thus

$$
P_{j} Q_{j} \sim \sum_{i=0}^{\infty} u_{i}^{\prime} j^{-3-i} \text { as } j \rightarrow \infty .
$$

Next, by the induction hypothesis we have

$$
R_{j} \equiv \theta_{2 n}^{(j+1)}-A \sim \zeta^{j} \sum_{i=0}^{\infty} r_{i} j^{\gamma_{n}-i} \text { as } j \rightarrow \infty
$$

and

$$
S_{j} \equiv \Delta \theta_{2 n-1}^{(j+1)} \sim \zeta^{-j} \sum_{i=0}^{\infty} s_{i} j^{-\gamma_{n-1}-i} \text { as } j \rightarrow \infty
$$

and hence

$$
\Delta\left(R_{j} S_{j}\right) \sim \sum_{i=0}^{\infty} u_{i}^{\prime \prime} j^{\gamma_{n}-\gamma_{n-1}-i-1} \text { as } j \rightarrow \infty
$$


Consequently, by the induction hypothesis that $\gamma_{n}=\gamma_{n-1}-3-\mu_{n}$ for some nonnegative integer $\mu_{n}$, we have

$$
U_{j}=P_{j} Q_{j}-\Delta\left(R_{j} S_{j}\right) \sim \sum_{i=0}^{\infty} u_{i} j^{-3-i} \text { as } j \rightarrow \infty .
$$

In addition, since $\theta_{2 n+1}^{(j)}$ satisfies (3.9) by the induction hypothesis,

$$
V_{j} \equiv \Delta^{2} \theta_{2 n+1}^{(j)} \sim \zeta^{-j} \sum_{i=0}^{\infty} v_{i} j^{-\gamma_{n}-i} \text { as } j \rightarrow \infty, \quad v_{0}=\left(\zeta^{-1}-1\right)^{2} h_{n 0} \neq 0 .
$$

Combining (3.19) and (3.20) in (3.12), we obtain

$$
\theta_{2 n+2}^{(j)}-A=\frac{U_{j}}{V_{j}} \sim \zeta^{j} \sum_{i=0}^{\infty} g_{i}^{\prime} j^{\gamma_{n}-3-i} \text { as } j \rightarrow \infty,
$$

which gives the desired result for $\theta_{2 n+2}^{(j)}$, namely,

$$
\theta_{2 n+2}^{(j)}-A \sim \zeta^{j} \sum_{i=0}^{\infty} g_{n+1, i} j^{\gamma_{n+1}-i} \text { as } j \rightarrow \infty, \quad g_{n+1,0} \neq 0,
$$

with $\gamma_{n+1}=\gamma_{n}-3-\mu_{n+1}$ for some nonnegative integer $\mu_{n+1}$.

As for $\theta_{2 n+3}^{(j)}$, we start with

$$
\theta_{2 n+3}^{(j)}=\theta_{2 n+1}^{(j+1)}+\frac{1}{\Delta \theta_{2 n+2}^{(j)}} .
$$

By the induction hypothesis we first have

$$
\theta_{2 n+1}^{(j+1)} \sim \zeta^{-j} \sum_{i=0}^{\infty} h_{i}^{\prime} j^{-\gamma_{n}-i} \text { as } j \rightarrow \infty, \quad h_{0}^{\prime}=h_{n 0} / \zeta \neq 0 .
$$

Next, by (3.22) we have

$$
\frac{1}{\Delta \theta_{2 n+2}^{(j)}} \sim \zeta^{-j} \sum_{i=0}^{\infty} h_{i}^{\prime \prime} j^{-\gamma_{n+1}-i} \text { as } j \rightarrow \infty, \quad h_{0}^{\prime \prime}=1 /\left[(\zeta-1) g_{n+1,0}\right] \neq 0 .
$$

Combining (3.24) and (3.25) in (3.23), and recalling that $\gamma_{n+1}-\gamma_{n}=-3-\mu_{n+1} \leq$ -3 , we first have the asymptotic equality

$$
\theta_{2 n+3}^{(j)} \sim \frac{1}{\Delta \theta_{2 n+2}^{(j)}} \text { as } j \rightarrow \infty
$$

and, as a result,

$$
\theta_{2 n+3}^{(j)} \sim \zeta^{-j} \sum_{i=0}^{\infty} h_{n+1, i} j^{-\gamma_{n+1}-i} \text { as } j \rightarrow \infty, \quad h_{n+1,0}=h_{0}^{\prime \prime},
$$

which is the desired result for $\theta_{2 n+3}^{(j)}$. This completes the proof of part (ii) of the theorem.

As the representation of $\theta_{2 n+2}^{(j)}$ given in (3.2) looks simpler than that in (3.1), one may wonder whether it is not easier to use (3.2) in the proof of Theorem 3.2 It turns out that the convergence result produced by (3.2) is inferior to that stated in Theorem 3.2. The representation in (3.2) turns out to be useful in the stability analysis that we present in the next section, however. 


\section{Stability}

An issue of major importance in convergence acceleration is that of stability. By studying this issue carefully, we may understand why convergence acceleration methods behave the way they do, and we can also design ways of improving the convergence of these methods, at least in some cases. This has been done successfully in the papers [12] and [13] by the author in connection with the $d$-transformation of Levin and Sidi [6].

Our purpose now is to study the stability of the iterated $W$-transformation and the $\theta$-algorithm as these are applied to the sequences $\left\{A_{k}\right\}$ that have been treated in the previous sections. It turns out that both methods can be analyzed simultaneously with respect to stability.

From (1.15) and (1.14) we have

$$
\begin{gathered}
B_{n+1}^{(j)}=\lambda_{n}^{(j)} B_{n}^{(j+1)}+\mu_{n}^{(j)} B_{n}^{(j+2)}, \\
\lambda_{n}^{(j)}=-\frac{\Delta\left(1 / \Delta B_{n}^{(j)}\right)}{\Delta^{2}\left(1 / \Delta B_{n}^{(j)}\right)} \text { and } \mu_{n}^{(j)}=\frac{\Delta\left(1 / \Delta B_{n}^{(j+1)}\right)}{\Delta^{2}\left(1 / \Delta B_{n}^{(j)}\right)} .
\end{gathered}
$$

Similarly, from (3.2) we have

$$
\begin{gathered}
\theta_{2 n+2}^{(j)}=\lambda_{n}^{(j)} \theta_{2 n}^{(j+1)}+\mu_{n}^{(j)} \theta_{2 n}^{(j+2)} \\
\lambda_{n}^{(j)}=-\frac{\Delta \theta_{2 n+1}^{(j)}}{\Delta^{2} \theta_{2 n+1}^{(j)}} \text { and } \mu_{n}^{(j)}=\frac{\Delta \theta_{2 n+1}^{(j+1)}}{\Delta^{2} \theta_{2 n+1}^{(j)}} .
\end{gathered}
$$

If we denote both $B_{n}^{(j)}$ and $\theta_{2 n}^{(j)}$ by $Q_{n}^{(j)}$, we realize that (4.1) and (4.2) can be expressed as in

$$
Q_{n+1}^{(j)}=\lambda_{n}^{(j)} Q_{n}^{(j+1)}+\mu_{n}^{(j)} Q_{n}^{(j+2)} ; \quad \lambda_{n}^{(j)}+\mu_{n}^{(j)}=1,
$$

with the appropriate $\lambda_{n}^{(j)}$ and $\mu_{n}^{(j)}$.

Starting with the fact that $Q_{0}^{(j)}=A_{j}, j=0,1, \ldots$, we can easily see by induction that

$$
Q_{n}^{(j)}=\sum_{i=0}^{n} \gamma_{n i}^{(j)} A_{j+n+i} ; \quad \sum_{i=0}^{n} \gamma_{n i}^{(j)}=1
$$

for some $\gamma_{n i}^{(j)}$ that depend nonlinearly on $A_{k}, j \leq k \leq j+3 n$. As has been observed in the context of other extrapolation methods, the way $Q_{n}^{(j)}$ has been expressed in (4.4) is very suitable for the analysis of stability. The quantities of relevance to stability of the $Q_{n}^{(j)}$ are

$$
\Gamma_{n}^{(j)}=\sum_{i=0}^{n}\left|\gamma_{n i}^{(j)}\right|
$$

in the following sense. If $\bar{A}_{k}=A_{k}+\epsilon_{k}$ are the computed values of the $A_{k}$, then the application of the iterated $W$-transformation and the $\theta$-algorithm to $\left\{\bar{A}_{k}\right\}$ produces the approximations $\bar{Q}_{n}^{(j)}$ that are given by

$$
\bar{Q}_{n}^{(j)} \approx \sum_{i=0}^{n} \gamma_{n i}^{(j)} \bar{A}_{j+n+i}=Q_{n}^{(j)}+\sum_{i=0}^{n} \gamma_{n i}^{(j)} \epsilon_{j+n+i}
$$


for all practical purposes. (This is so since the $\gamma_{n i}^{(j)}$, even though they depend on the $A_{k}$, do not vary appreciably with small errors in the $A_{k}$.) Thus

$$
\left|\bar{Q}_{n}^{(j)}-Q_{n}^{(j)}\right| \lesssim \Gamma_{n}^{(j)} \epsilon, \quad \epsilon=\max \left\{\left|\epsilon_{k}\right|: j+n \leq k \leq j+2 n\right\} .
$$

That is, $\Gamma_{n}^{(j)}$ determines how close $\bar{Q}_{n}^{(j)}$ is to $Q_{n}^{(j)}$. For additional information on the meaning of $\Gamma_{n}^{(j)}$ we refer the reader to Section 4 of Sidi [14].

Obviously, when $\sup _{j} \Gamma_{n}^{(j)}=\infty$, the sequence $\left\{Q_{n}^{(j)}\right\}_{j=0}^{\infty}$ is unstable, and when $\sup _{j} \Gamma_{n}^{(j)}<\infty$, it is stable. Similarly, when $\sup _{n} \Gamma_{n}^{(j)}=\infty$, the sequence $\left\{Q_{n}^{(j)}\right\}_{n=0}^{\infty}$ is unstable, and when $\sup _{n} \Gamma_{n}^{(j)}<\infty$, it is stable.

Since $\sum_{i=0}^{n} \gamma_{n i}^{(j)}=1$ for both methods, as also follows immediately from Lemma 4.1 below, we have $\Gamma_{n}^{(j)} \geq 1$, and for good numerical stability we want $\Gamma_{n}^{(j)}$ to be as close to 1 as possible. In case of instability, we want $\Gamma_{n}^{(j)}$ to increase slowly as $j$ or $n \rightarrow \infty$.

Below we analyze $\Gamma_{n}^{(j)}$ for $j \rightarrow \infty$ just as we have analyzed the convergence of $\left\{Q_{n}^{(j)}\right\}_{j=0}^{\infty}$. The results of Sections 2 and 3 will be of use in this analysis.

We start with the following simple lemma.

Lemma 4.1. Let $P_{n}^{(j)}(z)=\sum_{i=0}^{n} \gamma_{n i}^{(j)} z^{i}$. Then

$$
P_{n+1}^{(j)}(z)=\lambda_{n}^{(j)} P_{n}^{(j+1)}(z)+\mu_{n}^{(j)} z P_{n}^{(j+2)}(z) .
$$

Proof. By (4.3) and (4.4) we have

$$
\sum_{i=0}^{n+1} \gamma_{n+1, i}^{(j)} A_{j+n+i+1}=\lambda_{n}^{(j)} \sum_{i=0}^{n} \gamma_{n i}^{(j+1)} A_{j+n+i+1}+\mu_{n}^{(j)} \sum_{i=0}^{n} \gamma_{n i}^{(j+2)} A_{j+n+i+2}
$$

from which

$$
\gamma_{n+1, i}^{(j)}=\lambda_{n}^{(j)} \gamma_{n i}^{(j+1)}+\mu_{n}^{(j)} \gamma_{n, i-1}^{(j+2)}, \quad i=0,1, \ldots, n+1,
$$

where we have defined $\gamma_{n,-1}^{(j)}=\gamma_{n, n+1}^{(j)}=0$. The result in (4.8) now follows from (4.10).

We next state a result on the behavior of $\lambda_{n}^{(j)}$ and $\mu_{n}^{(j)}$ as $j \rightarrow \infty$.

Lemma 4.2. (i) If $\left\{A_{k}\right\}$ is as in (1.1), then

$$
\lambda_{n}^{(j)} \sim \frac{j}{\gamma_{n}} \text { and } \mu_{n}^{(j)} \sim-\frac{j}{\gamma_{n}} \text { as } j \rightarrow \infty, \quad n \geq 0,
$$

with $\gamma_{k}$ as in part (i) of Theorem 2.2 or Theorem 3.2.

(ii) If $\left\{A_{k}\right\}$ is as in (1.2), then

$$
\lambda_{n}^{(j)} \sim \frac{\zeta}{\zeta-1} \text { and } \mu_{n}^{(j)} \sim-\frac{1}{\zeta-1} \text { as } j \rightarrow \infty, \quad n \geq 0 .
$$

(iii) If $\left\{A_{k}\right\}$ is as in (1.3), then

$$
\lambda_{n}^{(j)}=o(1) \text { and } \mu_{n}^{(j)} \sim 1 \text { as } j \rightarrow \infty, \quad n \geq 0 .
$$

The proof of this lemma can be achieved by employing the results of Theorems 2.2 and 3.2. We leave its details to the reader.

The main stability results are given in the next theorem. 
Theorem 4.3. (i) If $\left\{A_{k}\right\}$ is as in (1.1), then

$$
P_{n}^{(j)}(z) \sim\left(\prod_{k=0}^{n-1} \gamma_{k}\right)^{-1}(1-z)^{n} j^{n} \quad \text { and } \quad \Gamma_{n}^{(j)} \sim\left|\prod_{k=0}^{n-1} \gamma_{k}\right|^{-1}(2 j)^{n} \quad \text { as } j \rightarrow \infty .
$$

(ii) If $\left\{A_{k}\right\}$ is as in (1.2), then

$$
P_{n}^{(j)}(z) \sim\left(\frac{\zeta-z}{\zeta-1}\right)^{n} \text { and } \Gamma_{n}^{(j)} \sim\left(\frac{|\zeta|+1}{|\zeta-1|}\right)^{n} \text { as } j \rightarrow \infty .
$$

(Of course, when $\zeta=-1, \Gamma_{n}^{(j)} \sim 1$ as $j \rightarrow \infty$.)

(iii) If $\left\{A_{k}\right\}$ is as in (1.3), then

$$
P_{n}^{(j)}(z) \sim z^{n} \text { and } \Gamma_{n}^{(j)} \sim 1 \text { as } j \rightarrow \infty .
$$

Proof. The proofs of all three parts can be carried out by combining Lemma 4.1 and Lemma 4.2 and by using induction on $n$. We leave the details to the reader.

We can conclude from part (i) of Theorem 4.3 that neither the iterated $W$ transformation nor the $\theta$-algorithm is stable when applied to the logarithmic sequences in (1.1). They may achieve quite high numerical accuracy when $|\Im \gamma|$ is large, as in this case the factor $\left|\prod_{k=0}^{n-1} \gamma_{k}\right|^{-1}$ becomes small causing $\Gamma_{n}^{(j)}$ to be small, even though $\sup _{j} \Gamma_{n}^{(j)}=\infty$. This conclusion is the same as that reached in Sidi 14] for the Richardson extrapolation process.

From part (ii) of Theorem 4.3 we conclude that both methods are stable when applied to the linear sequences in (1.2). When $\zeta$ is close to 1 , however, $\Gamma_{n}^{(j)}$ may be very large even though $\sup _{j} \Gamma_{n}^{(j)}<\infty$. In order to stabilize the approximations $Q_{n}^{(j)}$ we propose to apply the methods to the subsequences $\left\{A_{s k}\right\}_{k=0}^{\infty}$ for some positive integer $s \geq 2$ such that $\zeta^{s} \neq 1$ and is farther from 1 than $\zeta$ is. For $\left\{A_{s k}\right\}_{k=0}^{\infty}$ we will have in this case

$$
A_{s n} \sim A+\left(\zeta^{s}\right)^{n} \sum_{i=0}^{\infty} \alpha_{i}^{\prime} n^{\gamma-i} \text { as } n \rightarrow \infty, \alpha_{0}^{\prime}=\alpha_{0} s^{\gamma} \neq 0 .
$$

An analogous strategy was already proposed in Sidi [12] in the application of the Levin-Sidi [6] $d$-transformation to power series and Fourier series and their generalizations.

Part (iii) of Theorem 4.3 implies that, when applied to the factorial sequences in (1.3), the methods are stable.

\section{COMPARISON With THE LEVIN $u$-Transformation}

The results we have obtained for the iterated $W$-transformation and the $\theta$ algorithm are similar to those pertaining to the Levin $u$-transformation that is defined in (1.18). We provide the latter here for comparison and completeness. Before we do that we also note that

$$
u_{n}^{(j)}=\sum_{i=0}^{n} \gamma_{n i}^{(j)} A_{j+i} ; \quad \gamma_{n i}^{(j)}=\frac{(-1)^{n-i}\left(\begin{array}{c}
n \\
i
\end{array}\right)(j+i)^{n-2} / \Delta A_{j+i}}{\Delta^{n}\left(j^{n-2} / \Delta A_{j}\right)}, \quad i=0,1, \ldots, n
$$


Theorem 5.1. (i) Let $\left\{A_{k}\right\}$ be as in (1.1), and let $\beta_{n+\mu}(\mu \geq 0)$ be the first nonzero $\beta_{i}$ with $i \geq n$ in (1.11). Then

$$
\begin{aligned}
& u_{n}^{(j)}-A \sim \sum_{i=0}^{\infty} w_{i} j^{\gamma-n-\mu-i} \quad \text { as } j \rightarrow \infty ; \quad w_{0}=(-1)^{n} \alpha_{0} \beta_{n+\mu} \gamma \frac{(\mu+1)_{n}}{(-\gamma)_{n}} \neq 0, \\
& P_{n}^{(j)}(z) \sim \frac{j^{n}}{(-\gamma)_{n}}(z-1)^{n} \text { and } \Gamma_{n}^{(j)} \sim \frac{(2 j)^{n}}{\left|(-\gamma)_{n}\right|} \text { as } j \rightarrow \infty .
\end{aligned}
$$

(ii) Let $\left\{A_{k}\right\}$ be as in (1.2), and let $\beta_{n-1+\mu}(\mu \geq 0)$ be the first nonzero $\beta_{i}$ with $i \geq n-1$ in (1.12). Then

$$
\begin{aligned}
& u_{n}^{(j)}-A \sim \zeta^{j} \sum_{i=0}^{\infty} w_{i} j^{\gamma-2 n+1-\mu-i} \quad \text { as } j \rightarrow \infty ; \quad w_{0}=\alpha_{0} \beta_{n-1+\mu} \frac{\zeta^{n}}{(\zeta-1)^{n-1}} \neq 0, \\
& P_{n}^{(j)}(z) \sim\left(\frac{\zeta-z}{\zeta-1}\right)^{n} \text { and } \Gamma_{n}^{(j)} \sim\left(\frac{|\zeta|+1}{|\zeta-1|}\right)^{n} \quad \text { as } j \rightarrow \infty
\end{aligned}
$$

(iii) Let $\left\{A_{k}\right\}$ be as in (1.3), and let $\beta_{n-1+\mu}(\mu \geq 0)$ be the first nonzero $\beta_{i}$ with $i \geq n-1$ in (1.13). Then

$$
\begin{gathered}
u_{n}^{(j)}-A \sim\left(\Delta A_{j+n}\right) \sum_{i=0}^{\infty} \delta_{i} j^{-n-r-i} \quad \text { as } j \rightarrow \infty \\
\delta_{0}=-\zeta \cdot(-r-1)_{n} \neq 0, n \leq r+1, \\
u_{n}^{(j)}-A \sim\left(\Delta A_{j+n}\right) \sum_{i=0}^{\infty} \delta_{i} j^{-2 n+1-\mu-i} \text { as } j \rightarrow \infty ; \\
\delta_{0}=(-1)^{n} \beta_{n-1+\mu}(\mu+1)_{n} \neq 0, n>r+1, \\
P_{n}^{(j)}(z) \sim z^{n} \text { and } \Gamma_{n}^{(j)} \sim 1 \text { as } j \rightarrow \infty .
\end{gathered}
$$

As can be seen from this theorem, the $u$-transformation accelerates the convergence of $\left\{A_{k}\right\}$ in all cases.

Since the approximation $u_{n}^{(j)}$ is determined from $A_{k}, j \leq k \leq j+n+1$, and the approximation $Q_{n}^{(j)}\left(B_{n}^{(j)}\right.$ or $\left.\theta_{2 n}^{(j)}\right)$ is determined from $A_{k}, j \leq k \leq j+3 n$, we should compare the sequences $\left\{u_{3 n-1}^{(j)}\right\}_{j=0}^{\infty}$ and $\left\{Q_{n}^{(j)}\right\}_{j=0}^{\infty}$. From previous results we have, for $j \rightarrow \infty$,

$$
\begin{aligned}
& \left.Q_{n}^{(j)}-A=O\left(j^{\gamma-2 n}\right) \quad \text { and } \quad u_{3 n-1}^{(j)}-A=O\left(j^{\gamma-3 n+1}\right) \quad \text { (for (1.1) }\right) \text {, } \\
& Q_{n}^{(j)}-A=O\left(\zeta^{j} j^{\gamma-3 n}\right) \quad \text { and } \quad u_{3 n-1}^{(j)}-A=O\left(j^{\gamma-6 n+3}\right) \quad \text { (for (1.21)), } \\
& Q_{n}^{(j)}-A=O\left(\frac{\zeta^{j}}{(j !)^{r}} j^{\gamma-(3 r+2) n}\right) \text { and } \\
& \left.u_{3 n-1}^{(j)}-A=O\left(\frac{\zeta^{j}}{(j !)^{r}} j^{\gamma-(3 r+2) n-(4 n-r-3)}\right), n>r+1 \quad \text { for (1.3) }\right) \text {. }
\end{aligned}
$$




\section{REFERENCES}

[1] S. Bhowmick, R. Bhattacharya, and D. Roy. Iterations of convergence accelerating nonlinear transforms. Comput. Phys. Comm., 54:31-46, 1989. MR 90c:65005

[2] C. Brezinski. Accélération de suites à convergence logarithmique. C. R. Acad. Sci. Paris, 273 A:727-730, 1971. MR 46:4674

[3] J.E. Drummond. Summing a common type of slowly convergent series of positive terms. $J$. Austral. Math. Soc., Series B, 19:416-421, 1976. MR 57:18017

[4] W.B. Ford. Studies on Divergent Series and Summability. Chelsea, New York, 1960. MR 22:5839

[5] D. Levin. Development of non-linear transformations for improving convergence of sequences. Intern. J. Computer Math., B3:371-388, 1973. MR 50:11716

[6] D. Levin and A. Sidi. Two new classes of nonlinear transformations for accelerating the convergence of infinite integrals and series. Appl. Math. Comp., 9:175-215, 1981. Originally appeared as a Tel Aviv University preprint in 1975. MR 83d:65010

[7] S. Lubkin. A method of summing infinite series. J. Res. Nat. Bur. Standards, 48:228-254, 1952. MR 14:500g

[8] P. Sablonnière. Asymptotic behaviour of iterated modified $\Delta^{2}$ and $\theta_{2}$ transforms on some slowly convergent sequences. Numer. Algorithms, 3:401-409, 1992. MR 94f:65009

[9] D. Shanks. Nonlinear transformations of divergent and slowly convergent sequences. J. Math. and Phys., 34:1-42, 1955. MR 16:961e

[10] A. Sidi. Convergence properties of some nonlinear sequence transformations. Math. Comp., 33:315-326, 1979. MR 81h:65003

[11] A. Sidi. Analysis of convergence of the T-transformation for power series. Math. Comp., 35:833-850, 1980. MR 83d:41039

[12] A. Sidi. Acceleration of convergence of (generalized) Fourier series by the $d$-transformation. Annals Numer. Math., 2:381-406, 1995. MR 96h:65005

[13] A. Sidi. Convergence analysis for a generalized Richardson extrapolation process with an application to the $d^{(1)}$-transformation on convergent and divergent logarithmic sequences. Math. Comp., 64:1627-1657, 1995. MR 96a:65009

[14] A. Sidi. The Richardson extrapolation process with a harmonic sequence of collocation points. SIAM J. Numer. Anal., 37:1729-1746, 2000. MR 2001e:65007

[15] D.A. Smith and W.F. Ford. Acceleration of linear and logarithmic convergence. SIAM J. Numer. Anal., 16:223-240, 1979. MR 82a:65012

[16] D.A. Smith and W.F. Ford. Numerical comparisons of nonlinear convergence accelerators. Math. Comp., 38:481-499, 1982. MR 83d:65019

[17] A.H. Van Tuyl. Acceleration of convergence of a family of logarithmically convergent sequences, Math. Comp. 65:229-246. 1994. MR 94i:40001

[18] P. Wynn. On a device for computing the $e_{m}\left(S_{n}\right)$ transformation. Mathematical Tables and Other Aids to Computation, 10:91-96, 1956. MR 18:801e

Computer Science Department, Technion - Israel Institute of Technology, Haifa 32000 , ISRAEL

E-mail address: asidi@cs.technion.ac.il 